A N N A L E S Annales de Bretagne et des Pays de l'Ouest

Anjou. Maine. Poitou-Charente. Touraine

$121-1 \mid 2014$

Varia

\title{
Petites patries dans la Grande Guerre
}

\section{Stéphane Tison}

\section{OpenEdition}

Journals

Édition électronique

URL : http://journals.openedition.org/abpo/2755

DOI : $10.4000 / a b p o .2755$

ISBN : 978-2-7535-3407-0

ISSN : 2108-6443

Éditeur

Presses universitaires de Rennes

Édition imprimée

Date de publication : 27 mars 2014

Pagination : 201-203

ISBN : 978-2-7535-3405-6

ISSN : 0399-0826

Référence électronique

Stéphane Tison, «Petites patries dans la Grande Guerre », Annales de Bretagne et des Pays de l'Ouest [En ligne], 121-1 | 2014, mis en ligne le 27 mars 2014, consulté le 23 septembre 2020. URL : http:// journals.openedition.org/abpo/2755; DOI : https://doi.org/10.4000/abpo.2755 
l'a bien montré Judith Lyon-Caen (La lecture et la vie. Les usages du roman au temps de Balzac, Paris, Tallandier, 2006) et qui eût peut-être permis de penser l'œuvre de Souvestre à l'aune d'une nouvelle cohérence. En résumé, D. Steel nous livre un travail utile mais qui n'épuise visiblement pas son sujet.

Jean LE BIHAN

Bourlet, Mickaël, Lagadec, Yann, Le Gall, Erwan, Petites patries dans la Grande Guerre, Rennes, PUR, coll. « Histoire ", 2013, 250 p.

"La petite patrie est un espace aimable et protecteur, intermédiaire entre la famille et la société, au sein duquel l'individu s'épanouit et se développe. Pour ce qu'elle évoque de quiétude maternelle, elle se distingue de la Grande Patrie, également féminine, certes, dans l'appellation, mais plus altière, plus guerrière, plus virile en dernière instance ", écrivait Anne-Marie Thiesse en décrivant la façon dont l'institution scolaire de la III république lisait ces deux représentations qui, liées entre elles dans l'éducation des enfants, pouvaient permettre une éducation civique dans le cadre d'un enseignement de masse (Ils apprenaient la France : l'exaltation des régions dans le discours patriotique, Paris, éd. de la MSH, 1997, p. 7-8).

Cet ouvrage publié sous la direction de Michaël Bourlet, Yann Lagadec et Erwan Le Gall, livre une réflexion originale sur une étape de l'émergence de la question régionale, celle de la Grande Guerre, pendant laquelle le lien entre petite patrie et grande patrie est particulièrement questionné. Dans une introduction programmatique, les auteurs rappellent que la région demeure un angle mort de la recherche et inscrivent leur réflexion dans la continuité des travaux d'Anne-Marie Thiesse, JeanFrançois Chanet, Christian Bougeard. L'approche régionale s'avère riche d'enseignements car elle décentre le regard en permettant de quitter une lecture uniquement nationale, globale, traitant des phénomènes majeurs étudiés jusque-là. Les auteurs reviennent à juste titre sur la définition même des contours d'une région au début du $\mathrm{xx}^{\mathrm{e}}$ siècle. Il ne s'agit pas de travailler sur des " pays " ni sur des régions qui seraient calqués sur un schéma administratif très contemporain, Les vestiges culturels des anciennes " provinces » demeurent toutefois dans l'identité des corps d'armée dont l'assise territoriale, la région militaire, recoupe partiellement des découpages administratifs de l'Ancien Régime. Un sentiment d'appartenance précédant souvent le sentiment national se maintient en effet dans l'usage des patois, dans la chanson, la toponymie, la mémoire. Les auteurs font émerger dès lors l'idée d'identités territoriales, ne correspondant à aucune entité administrative, mais vécues comme une entité culturelle.

L'interrogation porte sur diverses échelles : si la part belle est accordée au cas breton, le but de l'ouvrage est d'engager une comparaison intra nationale, avec une étude sur le Nord (Mikael Bourlet), l'Alsace-Lorraine (Raphaël Georges), sur les territoires à l'identité moins marquée comme la Basse-Normandie (Jérémie Hallais); la troisième partie, comprenant des contributions sur le Québec (Carl Pépin) et la Nouvelle Zélande (Nathalie Philippe), invite à une comparaison internationale sur les minorités d'Autriche-Hongrie, d'Italie, ou encore la question irlandaise.

Outre les apports au débat, les travaux proposés offrent un point de vue méthodologique très enrichissant. Depuis l'étude quantitative inspirée des travaux de Jules Maurin, réalisée avec une grande précision par Jérémie Hallais sur les conscrits de Granville, jusqu'à l'analyse des images issues des collections de l'ECPAD (Établissement de communication et de production audiovisuelle de la Défense) 
effectuée par Véronique Goloubinoff et David Sbrava, en passant par l'histoire culturelle à travers l'étude des usages du vocabulaire régionaliste par Odile Roynette ou l'analyse d'Emmanuelle Cronier des ressorts culturels du moral des troupes alliées. Soulignons notamment l'originalité de la contribution d'Erwann Le Gall sur le $47^{\mathrm{e}} \mathrm{RI}$ de Saint Malo qui propose une méthodologie très intéressante mettant en valeur des sources récemment rendues accessibles par leur mise en ligne sur internet et que nombre de chercheurs pourront mettre à profit : les fiches des soldats morts pour la France, les journaux de marche et opération et les historiques régimentaires (à consulter sur le site du Ministère de la Défense, Mémoire des hommes : http:// www.memoiredeshommes.sga.defense.gouv.fr). Toutefois, on attendrait davantage de réflexions méthodologiques sur les modalités d'une comparaison internationale seulement esquissée dans ce volume.

Plusieurs contributions montrent que le fait régional s'avère invisible au cours de la période. L'absence d'un sentiment d'appartenance régionale véritablement saillant pendant la période est due notamment à l'emboîtement des identités mis en œuvre depuis deux générations au moins par l'expérience de la conscription. Au cours des années de service militaire, l'appartenance locale, l'identité régionale, l'inscription de l'individu dans la nation, se mêlent et se trouvent subsumées par une identité de génération, celle de " la classe ". Il n'est alors pas étonnant que l'argot des tranchées n'intègre finalement qu'à la marge les dialectes locaux, comme le souligne $\mathrm{O}$. Roynette. L'incorporation des individus est aussi linguistique : elle favorise en effet une acculturation militaire par l'usage d'un vocabulaire moderne, qui n'empêche pas l'existence d'un bilinguisme, qui facilite l'intégration du conscrit puis du combattant au groupe, et qui permet d'éviter les moqueries tout en préservant son identité locale ou régionale. Toutefois, peu de lettres sont écrites en breton pendant la guerre, celle-ci ne suscitant pas, dans l'écrit ou par la parole, de retour au sentiment d'appartenance régionale. Cette identité ténue pourrait se réduire même du fait que le recrutement régional s'amenuise au fil du conflit. L'étude des effectifs du $47^{\mathrm{e}} \mathrm{RI}$ régiment de Saint-Malo par E. Le Gall montre que $90 \%$ des recrues viennent de Saint-Malo en 1914 tandis que ce chiffre tombe à moins de 50 \% à la fin de la guerre. Pour autant, les Malouins considèrent toujours cette unité comme le régiment de la ville, et l'affirment encore lors du retour des troupes sur les banderoles et dans les discours. L'auteur le souligne lui-même : il n'est guère possible malheureusement de savoir comment réagirent les non Malouins de ce régiment.

Si l'absence de revendication d'une appartenance régionale est évidente, les identités territoriales se maintiennent culturellement, à travers la persistance et l'usage de traditions culinaires par exemple. Malgré une francisation certaine, persiste une identité territoriale sur un mode folklorique, montrant les solidarités de ceux qui sont originaires des mêmes territoires, qui partagent des traditions gustatives, musicales, des parlers locaux. De telles solidarités jouent un rôle dans l'endurance des combattants : le support identitaire constitue assurément un moyen de traverser les moments éprouvants, sans pour autant se suffire à elle-même. Dès 1914, elle est associée à des niveaux plus larges (nation) ou plus petits (petites patries). Ce sont des " communautés imaginaires " qui sont fondées par l'expérience de guerre. En effet, le brassage de millions d'hommes contribue en retour à cristalliser des stéréotypes en gestation avant 1914 à travers l'apparition d'images relevant du folklore. Les Bretons sont ainsi présentés comme courageux, vertueux, tandis que les Méridionaux sont décrits comme impressionnables. Images contradictoires puisque les Bretons comme les Polonais sont vus comme très attachés à la religion, sans toutefois dédaigner la bouteille. L'histoire des régiments après guerre joue un rôle dans la façon de diffuser et figer ces stéréotypes. 
Au croisement de la nation et des petites patries, c'est bien au cœur des unités combattantes, des groupes primaires de combattants, d'unités comme le régiment, que se redessine le lien identitaire, et sans doute faut-il insister sur ce point. Si la référence est faite au "pays ", c'est pour se retrouver entre soi, face à l'adversité. C'est là une stratégie de survie, faisant écho en quelque sorte à la confrontation, à la dureté des temps, et à la violence du combat. Sans le dire clairement, les contributeurs de cet ouvrage font apparaître ce que nous pourrions appeler une « identité de situation ", liée à l'expérience de guerre, qui perdura au sortir du conflit lorsque les survivants et les endeuillés voulurent donner sens à leur souffrance pour traverser traumatismes et deuils. La référence identitaire se mêle alors à la commémoration. Le débat sur les pertes à l'échelle nationale et régionale, le besoin de définir et de savoir qui a le plus souffert constituent ainsi une justification des pertes, un moyen de faire son deuil en l'inscrivant dans cet ancrage national, tout en constituant le mode d'expression d'une souffrance particulière. Le lien entre le deuil de masse et la résurgence de demandes régionalistes paraît essentiel et nécessite encore d'être mesuré. Les revendications sont plus fortes pendant le conflit dans des territoires où la demande de reconnaissance est plus grande : c'est le cas du Québec où le sentiment d'appartenance passe par le refus de la conscription considérée comme une nouvelle pression du gouvernement britannique. C'est le cas également des Néo-Zélandais qui s'emparent $a$ contrario de l'enjeu guerrier pour affirmer leur existence en tant que nation et dépasser définitivement le statut de " région coloniale " lointaine à la périphérie de l'empire.

Au final, cet ouvrage collectif présente une façon renouvelée de faire une histoire de l'arrière délaissée depuis quelques décennies. La question de l'appartenance culturelle s'avère d'autant plus pertinente à l'heure où les collectivités territoriales vont contribuer largement à l'organisation du centenaire de la Grande Guerre. Les usages mémoriels de 14-18 donneront à cette occasion une idée du maintien ou de l'effacement de ces identités territoriales, sujet d'étude passionnant pour les sociologues d'aujourd'hui et les historiens à venir.

Stéphane Tison

Lagadec, Yann, Meuret, Jean-Claude, Rannou, Yves, Une entrée en guerre. Chelun, village breton (1914-1915), Rennes, Société archéologique et historique d'Ille-etVilaine, 2013, 88 p.

En publiant ce livre sur l'entrée en guerre de Chelun dès 2013, la Société archéologique et historique d'Ille-et-Vilaine inaugure et anticipe, du moins à l'échelle locale, le flot de publications qui devrait accompagner le centenaire de la Première Guerre mondiale. L'ouvrage, de dimension modeste - 88 pages -, se compose de deux parties indépendantes qui mettent chacune en lumière une source éclairant le basculement dans la guerre de cette commune rurale.

Le premier document présenté, et celui autour duquel s'est articulé ce projet d'édition, est le journal de François Louvel, instituteur public et secrétaire de mairie de Chelun, où il fut nommé en 1913. Pendant les trois mois qui précédèrent sa mobilisation, de juillet à octobre 1914, il tint une chronique - quotidienne à partir de fin août - sur la vie de cette commune d'environ 600 habitants du canton de La Guerche. Le texte, intégralement reproduit, est remarquablement annoté et introduit par Yann Lagadec et Yves Rannou, qui donnent au lecteur toutes les clés nécessaires pour comprendre le document. 